Supporting Information

\title{
Mixed Valence and Superconductivity in Perovskite Antimonates
}

Minu Kim ${ }^{1, *}$, Steffen Klenner ${ }^{2}$, Graham M. McNally ${ }^{1}$, Jürgen Nuss ${ }^{1}$, Alexander Yaresko ${ }^{1}$, Ulrich

Wedig $^{1}$, Reinhard K. Kremer ${ }^{1}$, Rainer Pöttgen ${ }^{2, *}$, and Hidenori Takagi ${ }^{1,3}$

${ }^{1}$ Max Planck Institute for Solid State Research, Heisenbergstrasse 1, 70569 Stuttgart, Germany.

${ }^{2}$ Institut für Anorganische und Analytische Chemie, Universität Münster, Corrensstrasse 30, 48149 Münster, Germany.

${ }^{3}$ Department of Physics, University of Tokyo, Bunkyo-ku, Hongo 7-3-1, Tokyo 113-0033, Japan. 
(a)

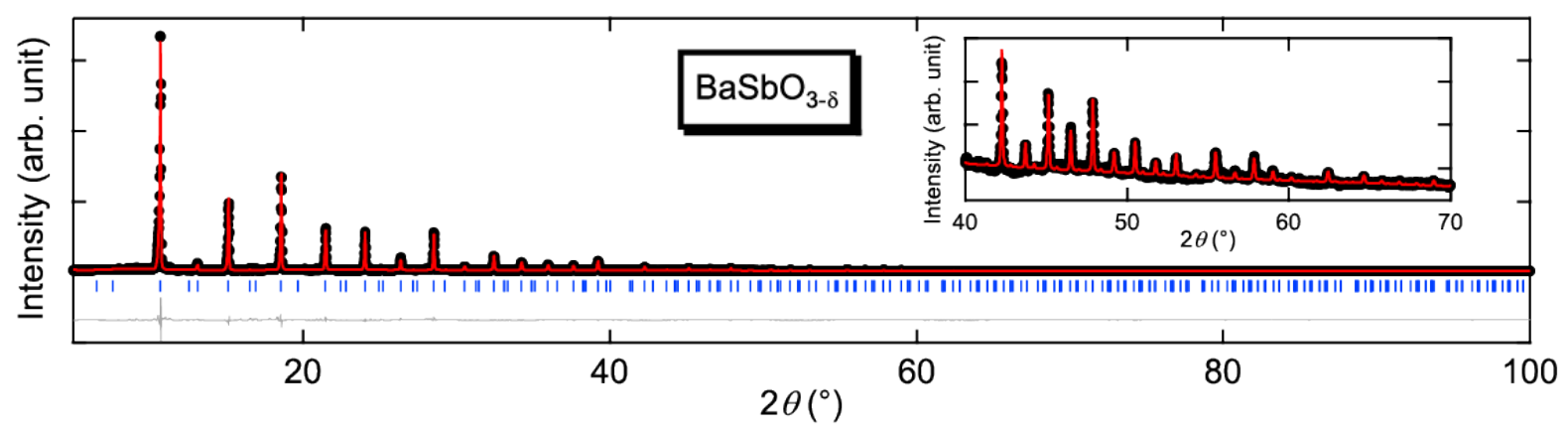

(b)

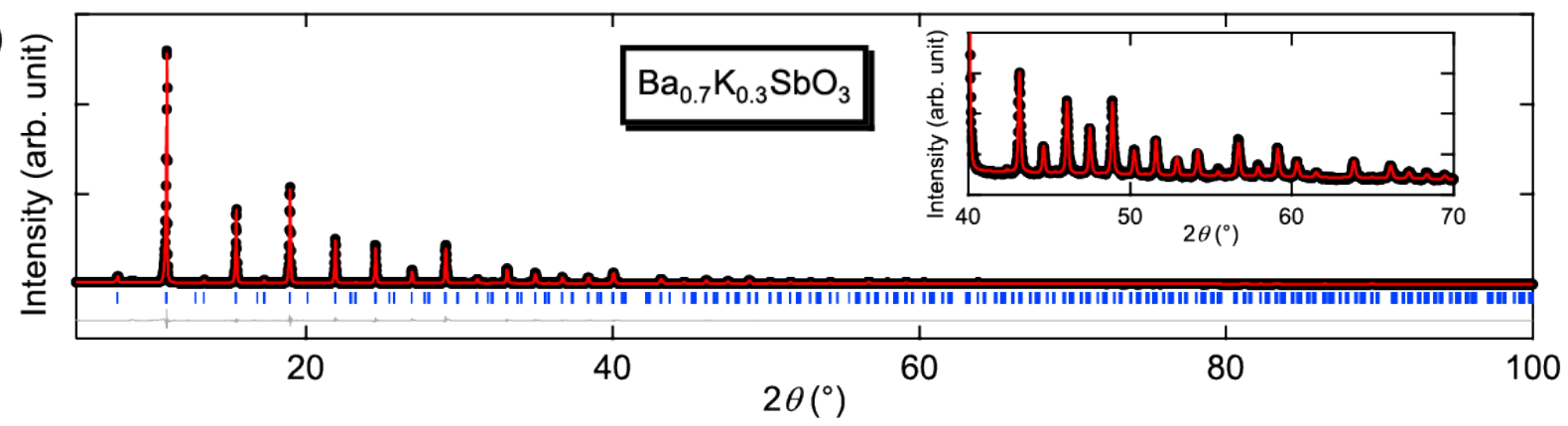

(c)

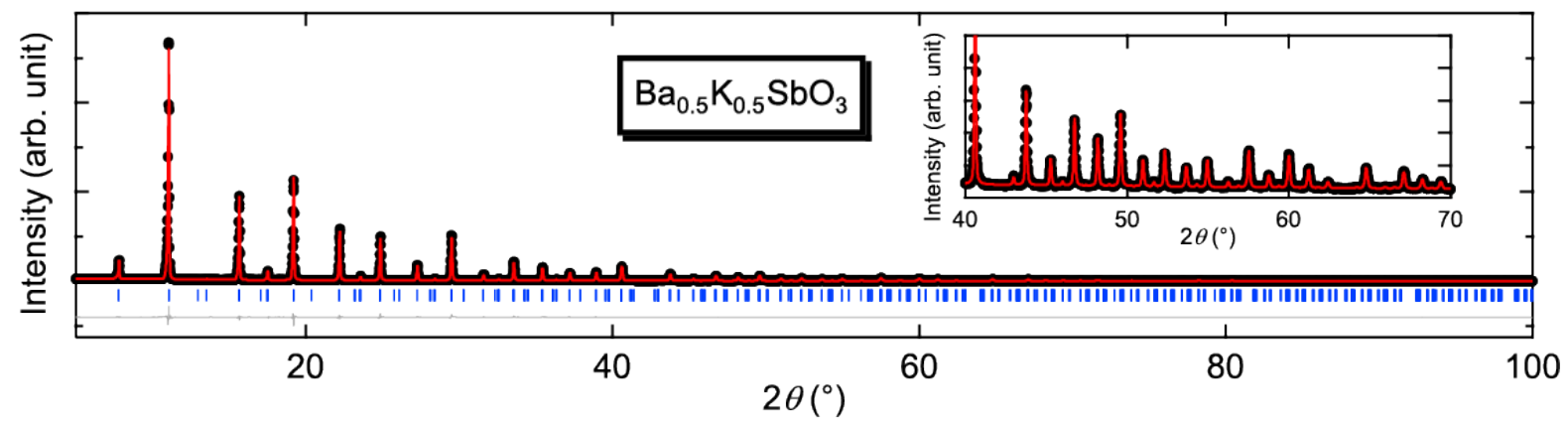

(d)

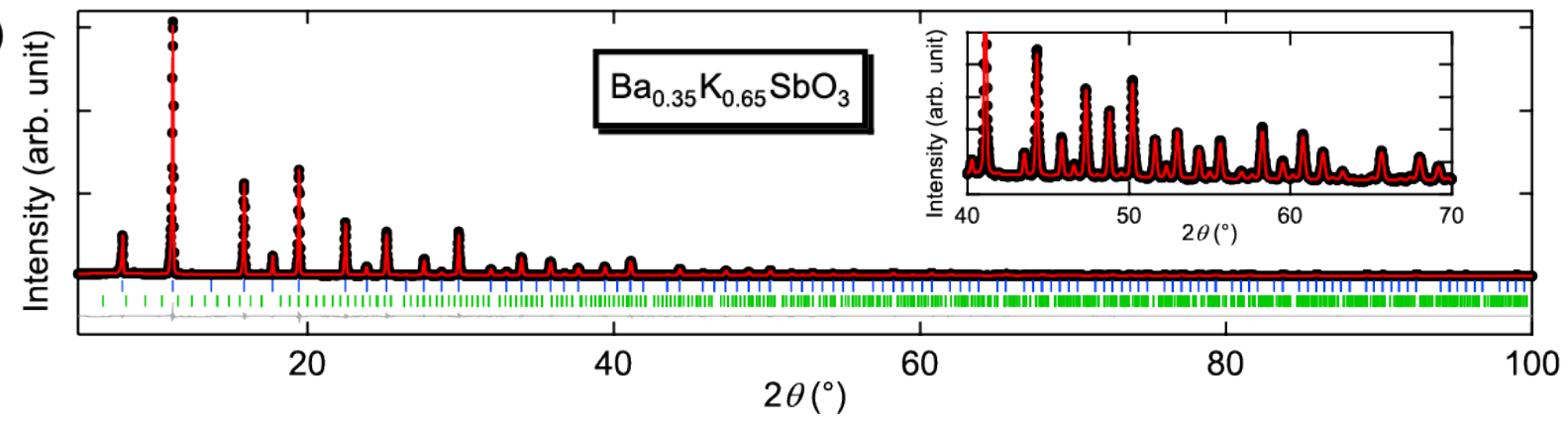

Figure S1. Rietveld refinement of $\mathrm{Ba}_{1-x} \mathrm{~K}_{x} \mathrm{SbO}_{3}$ samples measured via $\mathrm{Ag} K_{\alpha 1}$ source $(\lambda=0.5594 \AA)$ at $300 \mathrm{~K}$. Black circles: experimental data, red line: simulation, blue stick: Bragg positions of main phase, green stick: Bragg positions of secondary phase $\left(\mathrm{KSbO}_{3}\right)$, grey line: difference between experimental data and simulation. Inset shows reflections at a high $2 \theta$ range. 
$\mathrm{BaSbO}_{3-\delta}, F m \overline{3} m, Z=8, a=8.5023(2) \AA, R_{\mathrm{wp}}=9.82 \%$

\begin{tabular}{|c|c|c|c|c|c|c|c|c|c|c|}
\hline \multirow[t]{2}{*}{ Name } & \multirow[t]{2}{*}{ Type } & \multirow[t]{2}{*}{$x$} & \multirow[t]{2}{*}{$y$} & \multirow[t]{2}{*}{$z$} & \multirow[t]{2}{*}{ fraction } & \multicolumn{2}{|c|}{ site symmetry } & \multirow[t]{2}{*}{ multiplicity } & \multirow{2}{*}{$\begin{array}{c}\text { thermal } \\
\text { displacement }\end{array}$} & \multirow[t]{2}{*}{$B_{\text {iso }}\left(\AA^{2}\right)$} \\
\hline & & & & & & $\begin{array}{l}\text { Hermann- } \\
\text { Mauguin }\end{array}$ & Schönflies & & & \\
\hline $\mathrm{Ba}$ & $\mathrm{Ba}+2$ & 0.25 & 0.25 & 0.25 & 1 & $\overline{4} 3 m$ & $T_{d}$ & 8 & Isotropic & $1.74(5)$ \\
\hline $\mathrm{Sb} 1$ & $\mathrm{Sb}+3$ & 0 & 0 & 0 & 1 & $m \overline{3} m$ & $O_{h}$ & 4 & Isotropic & $1.01(6)$ \\
\hline $\mathrm{Sb} 2$ & $\mathrm{Sb}+5$ & 0.5 & 0.5 & 0.5 & 1 & $m \overline{3} m$ & $O_{h}$ & 4 & Isotropic & $2.29(10)$ \\
\hline $\mathrm{O}$ & $\mathrm{O}-2$ & 0.2609 & 0 & 0 & 0.962 & $4 m \cdot m$ & $C_{4 v}$ & 24 & Isotropic & $3.70(19)$ \\
\hline
\end{tabular}

$\mathrm{Ba}_{0.7} \mathrm{~K}_{0.3} \mathrm{SbO}_{3}, I 4 / m c m, Z=4, a=5.8966(2) \AA, c=8.3265(4) \AA, R_{\mathrm{wp}}=6.99 \%$

\begin{tabular}{|c|c|c|c|c|c|c|c|c|c|c|}
\hline \multirow[t]{2}{*}{ Name } & \multirow[t]{2}{*}{ Type } & \multirow[t]{2}{*}{$x$} & \multirow[t]{2}{*}{$y$} & \multirow[t]{2}{*}{$z$} & \multirow[t]{2}{*}{ fraction } & \multicolumn{2}{|c|}{ site symmetry } & \multirow[t]{2}{*}{ multiplicity } & \multirow{2}{*}{$\begin{array}{c}\text { thermal } \\
\text { displacement }\end{array}$} & \multirow[t]{2}{*}{$B_{\text {iso }}\left(\AA^{2}\right)$} \\
\hline & & & & & & $\begin{array}{l}\text { Hermann- } \\
\text { Mauguin }\end{array}$ & Schönflies & & & \\
\hline $\mathrm{Ba}$ & $\mathrm{Ba}+2$ & 0 & 0.5 & 0.75 & $0.695(3)$ & $\overline{4} 2 m$ & $D_{2 d}$ & 4 & Isotropic & $0.85(2)$ \\
\hline K & $\mathrm{K}+1$ & 0 & 0.5 & 0.75 & $0.305(3)$ & $\overline{4} 2 m$ & $D_{2 d}$ & 4 & Isotropic & $0.85(2)$ \\
\hline $\mathrm{Sb}$ & $\mathrm{Sb}+5$ & 0 & 0 & 0 & 1 & $4 / m$. & $C_{4 h}$ & 4 & Isotropic & $0.73(2)$ \\
\hline $\mathrm{O} 1$ & $\mathrm{O}-2$ & 0 & 0 & 0.25 & 1 & 422 & $D_{4}$ & 4 & Isotropic & $1.70(7)$ \\
\hline $\mathrm{O} 2$ & $\mathrm{O}-2$ & 0.25 & 0.75 & 0 & 1 & $m .2 \mathrm{~m}$ & $C_{2 v}$ & 8 & Isotropic & $1.70(7)$ \\
\hline
\end{tabular}

$\mathrm{Ba}_{0.5} \mathrm{~K}_{0.5} \mathrm{SbO}_{3}, I 4 / m c m, Z=4, a=5.8179(1) \AA, c=8.2206(3) \AA, R_{\mathrm{wp}}=5.77 \%$

\begin{tabular}{|c|c|c|c|c|c|c|c|c|c|c|}
\hline \multirow[t]{2}{*}{ Name } & \multirow[t]{2}{*}{ Type } & \multirow[t]{2}{*}{$x$} & \multirow[t]{2}{*}{$y$} & \multirow[t]{2}{*}{$z$} & \multirow[t]{2}{*}{ fraction } & \multicolumn{2}{|c|}{ site symmetry } & \multirow[t]{2}{*}{ multiplicity } & \multirow{2}{*}{$\begin{array}{c}\text { thermal } \\
\text { displacement }\end{array}$} & \multirow[t]{2}{*}{$B_{\text {iso }}\left(\AA^{2}\right)$} \\
\hline & & & & & & $\begin{array}{l}\text { Hermann- } \\
\text { Mauguin }\end{array}$ & Schönflies & & & \\
\hline $\mathrm{Ba}$ & $\mathrm{Ba}+2$ & 0 & 0.5 & 0.75 & $0.489(2)$ & $\overline{4} 2 m$ & $D_{2 d}$ & 4 & Isotropic & $0.55(1)$ \\
\hline K & $\mathrm{K}+1$ & 0 & 0.5 & 0.75 & $0.511(2)$ & $\overline{4} 2 m$ & $D_{2 d}$ & 4 & Isotropic & $0.55(1)$ \\
\hline $\mathrm{Sb}$ & $\mathrm{Sb}+5$ & 0 & 0 & 0 & 1 & $4 / m$. & $C_{4 h}$ & 4 & Isotropic & $0.44(1)$ \\
\hline $\mathrm{O} 1$ & $\mathrm{O}-2$ & 0 & 0 & 0.25 & 1 & 422 & $D_{4}$ & 4 & Isotropic & $0.99(4)$ \\
\hline $\mathrm{O} 2$ & $\mathrm{O}-2$ & 0.25 & 0.75 & 0 & 1 & $m .2 \mathrm{~m}$ & $C_{2 v}$ & 8 & Isotropic & $0.99(4)$ \\
\hline
\end{tabular}


$\mathrm{Ba}_{0.35} \mathrm{~K}_{0.65} \mathrm{SbO}_{3}, P m \overline{3} m, Z=1, a=4.0643(1) \AA, R_{\mathrm{wp}}=5.92 \%$

\begin{tabular}{|c|c|c|c|c|c|c|c|c|c|c|}
\hline Name & Type & $x$ & $y$ & $z$ & fraction & \multicolumn{2}{|c|}{ site symmetry } & multiplicity & $\begin{array}{c}\text { thermal } \\
\text { displacement }\end{array}$ & $B_{\text {iso }}\left(\AA^{2}\right)$ \\
\cline { 6 - 9 } & & & & & & $\begin{array}{c}\text { Hermann- } \\
\text { Mauguin }\end{array}$ & Schönflies & & & \\
\hline $\mathrm{Ba}$ & $\mathrm{Ba}+2$ & 0.5 & 0.5 & 0.5 & $0.335(1)$ & $m \overline{3} m$ & $O_{h}$ & 1 & Isotropic & $0.44(1)$ \\
\hline $\mathrm{K}$ & $\mathrm{K}+1$ & 0.5 & 0.5 & 0.5 & $0.665(1)$ & $m \overline{3} m$ & $O_{h}$ & 1 & Isotropic & $0.44(1)$ \\
\hline $\mathrm{Sb}$ & $\mathrm{Sb}+5$ & 0 & 0 & 0 & 1 & $m \overline{3} m$ & $O_{h}$ & 1 & Isotropic & $0.32(1)$ \\
\hline $\mathrm{O}$ & $\mathrm{O}-2$ & 0 & 0 & 0.5 & 1 & $4 / m m . m$ & $D_{4 h}$ & 3 & Isotropic & $0.68(3)$ \\
\hline
\end{tabular}

Table S1. Refined structural parameters of $\mathrm{Ba}_{1-x} \mathrm{~K}_{x} \mathrm{SbO}_{3}$ samples at $300 \mathrm{~K}$. The position and fraction of oxygen were taken from neutron diffraction results, which will be published elsewhere. 
(a)

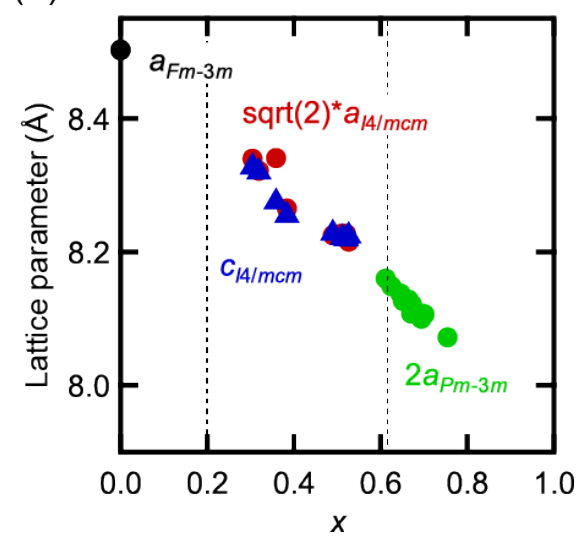

(b)

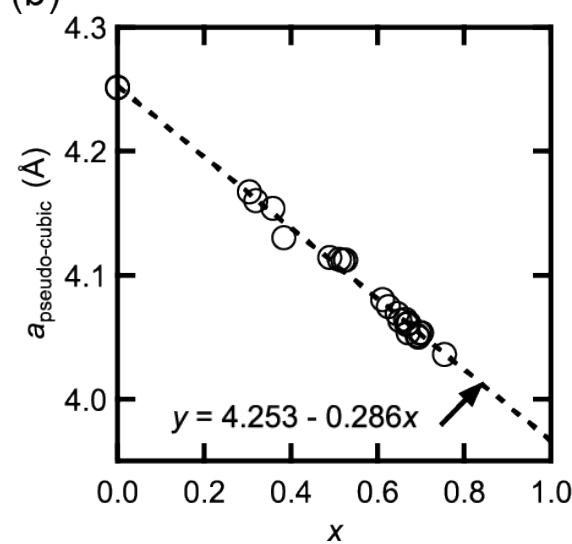

Figure S2. The evolution of (a) lattice parameter and (b) pseudo-cubic lattice parameter as a function of $x$ at $300 \mathrm{~K}$. 